REVISTA MATEMÁTICA de Ia

Universidad Complutense de Madrid

Volumen 10, número Suplementario: 1997

http://dx.doi.org/10.5209/rev_REMA.1997.v10.17335

\title{
Inner metric properties of 2-dimensional semi-algebraic sets.
}

\author{
L. BRÖCKER, M. KUPPE and W. SCHEUFLER
}

\begin{abstract}
We consider 2- dimensional semialgebraic topological manifolds from the differentialgeometric point of view. Curvatures at singularities are defined and a Gauss-Bonnet formula holds. Moreover, Aleksandrov's axioms for an intrinsic geometry of surfaces are fullfilled.
\end{abstract}

\section{Introduction}

A metric $d$ on a space $X$ is called inner metric, if for all $x, y \in X$ one has $d(x, y)=\inf (l(\gamma))$. Here the infimum is taken over all rectificable curves $\gamma$ connecting $x$ with $y$ and $l(\gamma)$ is the length of $\gamma$ (we set $d(x, y)=\infty$ if $x$ and $y$ are in different path components). We want to study semialgebraic sets from the inner metric point of view, and to start with, we consider sets of pure dimension 2 . We will consider curvatures along 1-dimensional singular subsets and at very singular points. Let us give some examples:

Example 1.1. Consider the surface $Q$ of the standard cube, being imbedded in $\mathbb{R}^{3}$. Then $Q$ is locally euclidean at all points except for the 8 vertices (even at the edges). The total angle at each vertex is $\frac{3}{2} \pi$. So we count a curvature $\frac{1}{2} \pi$, concentrated at each vertex. For the total curvature we get $8 \cdot \frac{1}{2} \pi=4 \pi=2 \pi \chi(Q)$ where $\chi$ is the Euler characteristic.

Mathematics Subject Classification: 14P10-14P99

Servicio Publicaciones Univ. Complutense. Madrid, 1997. 
Example 1.2. Consider a 2-dimensional cone $C \subset \mathbb{R}^{3}$ with the bottom as in the figure below which one gets by gluing, say, a unit disc and a half disc of radius 2 along the circle and halfcircle and the edges a respectively. This time we get a total angle of $\pi$ at $p$, hence the curvature $\pi$, and along the 1-dimensional singular edge we get the curvature $\int_{0}^{2 \pi}\left(\frac{1}{2}+1\right) d t=3 \pi$. At the smooth points $C$ is euclidean. So, as total curvature, we get again $4 \pi=2 \pi \chi(C)$.
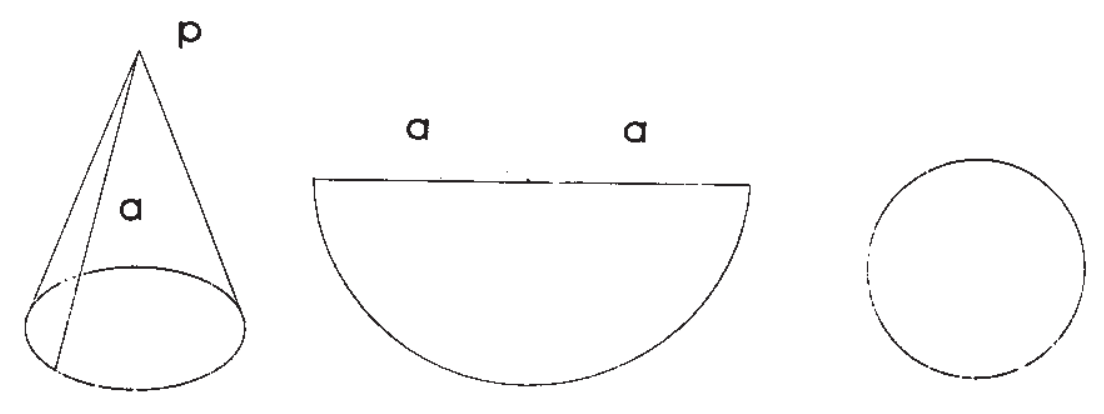

Example 1.3. Consider in $\mathbb{R}^{3}$ the surfaces

$$
S=\left\{x \in \mathbb{R}^{3} \mid x_{3}^{2}=\left(1-\sqrt{x_{1}^{2}+x_{2}^{2}}\right)^{3}, 0 \leq x_{1}^{2}+x_{2}^{2} \leq 1\right\}
$$

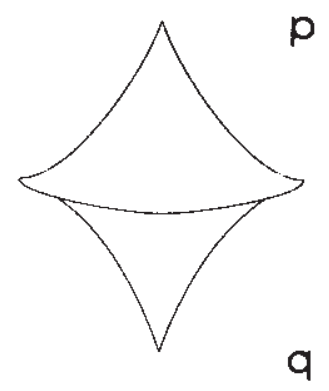

Here the total angle at the points $p$ and $q$ is $\frac{4}{\sqrt{13}} \pi$, the total curvature along the singular edge $S^{1}$ cannot be computed by usual formulas for curvatures of curves in Riemannian 2-manifolds, since one cannot differentiate sufficiently often. However, it will turn out, that the curvature along $S^{1}$ is $\int_{0}^{2 \pi} 2 d t=4 \pi$. The curvature at the smooth points is negative. 
In general, for any compact semi-algebraic 2-dimensional oriented manifold $S$ we expect a formula like

$$
\int_{S^{0}} K(x) d x+\int_{S^{1}} k(t) d t+\sum_{i=1}^{r} \kappa\left(p_{i}\right)=2 \pi \chi(S),
$$

where $S^{0}$ is the non-singular part of $S$ with Gauss-curvature $K$ and canonical measure $d x, k$ is the curvature along the 1-dimensional singular locus and $\kappa$ is the curvature, which is concentrated at finitetly many very singular points $p_{1}, \ldots, p_{r}$. We are going to explain, how $k$ and $\kappa$ can be defined. We will also investigate, how this fits into Aleksandrovs surface theory $[\mathrm{A}-\mathrm{Z}]$, and thus find a new class of models, for which Aleksandrovs axioms hold.

\section{Semi-algebraic geometry}

Let $S \subset \mathbf{R}^{n}$ be a smooth semi-algebraic 2 -manifold, endoved with the induced Riemannian metric. As before, we denote by $K$ the Gaubcurvature and by $d x$ the canonical measure on $S$.

Proposition 2.1. $\int_{S}|K(x)| d x<\infty$

In fact, much more is known: If $S^{d} \subset \mathbf{R}^{n}$ is a smooth semi-algebraic $d$-manifold, then $\int_{S}|\omega|$ is bounded by a function, which only depends on the complexity of a description for $S$, that is on $d, n$ and the number and degrees of the polynomials, which describe $S[\mathrm{Eu}]$. Here $\omega$ is the Gauß-Bonnet form according to the induced Riemannian metric. Note that $|\omega|$ is also defined if $S$ is non oriented. $\omega=0$ if $d$ is odd. Similary one has

Proposition 2.2. Let $S \subset \mathbb{R}^{n}$ be a smooth semi-algebraic 1-manifold. Then

$$
\int_{S}|k(t)| d t<\infty
$$

Here $k$ denotes the usual curvature for regular curves and $d t$ its length element. Again, the integral depends only on the complexity of describing equations for $S$. In fact, consider the semi-algebraic map

$$
\gamma: S \rightarrow S^{n-1} ; t \mapsto \frac{T_{t} S}{\left\|T_{t} S\right\|} \quad \text { (unit tangentvector) }
$$


Then $\int_{S}|k(t)| d t=$ length of $\gamma(S)$, and the right hand side can be estimated by a Cauchy-Crofton formula [Sa, Chap. 18, 1.].

Now assume that $S$ is a topological semi-algebraic 2-manifold with boundary $\partial S$. Let $S^{0}=S \backslash \partial S$ be non singular. Then, except for a finite set $S^{2} \subset \partial S$ at the points $y \in \partial S$ one has Nash-wings. That means the following:

One has a smooth neighbourhood $Y$ of $y$ in $\partial S$ and a tubular neighbourhood $T \rightarrow Y \times[0,1]$ around $Y$ together with a section $\sigma: Y \times[0,1[\rightarrow$ $T ;(y, t) \mapsto \sigma(y, t)$, so that the vanishing order of $\left.\frac{\partial}{\partial t} \sigma\right|_{0}$ is constant along $Y$. It follows, that the tangent plane map

$$
S^{0} \rightarrow G_{2 n} \quad ; \quad p \mapsto T_{p} S^{0}
$$

where $G_{2 n}$ is the Grassmann bundle, can be extended continuously from $S^{0}$ to $S \backslash S^{2}$. [B-C-R, Th. 9.6.13 and Th. 9.6.8]. In this situation $y$ is called a Nash-point. We also need the following

Lemma 2.3. Let $S \subset \mathbf{R}^{n}$ be a semi-algebraic topological 2-manifold. For $p \in S$ let $S(\rho, p)$ be the $n-1$ sphere with center $p$ and radius $\rho$. Let $S$ be stratified: $S=S^{0} \cup S^{1} \cup S^{2}, \operatorname{dim}\left(S^{i}\right)=2-i$ such that $S^{0}$ is smooth.

Then for all $\epsilon>0$ there exists $\delta>0$ such that for all $\delta$ with $0<\rho<\delta$ one has:

i) $S(\rho, p)$ intersects the strata $S^{i}, i=0,1,2$, transversally.

ii) For $q \in S(\rho, p) \cap S^{1}$ one has $\mid\langle X, Y>-1|<\epsilon$ where $X$ is unit tangent vector of $S^{1}$ at $q$ and $Y$ is unit normal vector on $S(\rho, p)$ at $q\left(S^{1}\right.$ intersects $S(\rho, p)$ nearly orthogonally. $X$ and $Y$ are defined up to a sign.)

iii) For $q \in S(\rho, p) \cap S^{0}$ one has $\mid\langle Y, N>-1|<\epsilon$ where again $Y$ is unit normal vector on $S(\rho, p)$ at $q$ and $N$ unit normal vector on $S(\rho, p) \cap S^{0}$ at $q$ (in the tangent space of $S^{0}$ at $q$ ).

Since we do not know a reference for this, we give a sketch of the proof: For $\epsilon>0$ it is easy to find a semi-algebraic stratification of $S$ such that on each 2-stratum the variation of the tangent space in the Grassmannian is less than $\epsilon$. Since statement ii) for 1-strata is rather obvious we get i) and iii) nearly automatically.

We are indebted to the referee for this argument. 


\section{Curvature and Gauß-Bonnet formula}

In the sequel we will have to consider curves and angles between curves on 2-manifolds (possibly with boundary). We will do this in various situations, for instance, in semi-algebraic topological 2-manifolds and in Aleksandrov surfaces. So this is just the general setting: A curve is always a simple continuous map $\gamma: I \rightarrow S$ where $I$ is an interval in $\mathbf{R}$ or $I$ is the unit circle. In the latter case $\gamma$ is called closed. The restriction of $\gamma$ to $I^{0}$ will be denoted by $\stackrel{\circ}{\gamma}$.

Let $p \in S$ and let $\gamma, \delta$ be curves with common initial point $p$ but no further common points. If $p \notin \partial S, \gamma$ and $\delta$ define two germs of regions, say $(\gamma, \delta)_{l}$ and $(\gamma, \delta)_{r}$ locally around $p$. If $S, \gamma$ and $\delta$ fullfill certain regularity conditions, there will exist section angles, corresponding to the two regions, which we denote by $\alpha_{l}(\gamma, \delta)$ and $\alpha_{r}(\gamma, \delta)$. In general, it can happen that $\alpha_{l}(\gamma, \delta)+\alpha_{r}(\gamma, \delta)=: \alpha(p) \neq 2 \pi$. This $\alpha(p)$ is called total angle around $p$. If $p \in \partial S$, there is only one section angle.

Similary, a curve $\gamma$ defines two sides and to each side corresponds a curvature $k_{l}$ or $k_{r}$ respectively of $\gamma$. (If $\gamma$ is closed, one has to assume that a neighbourhood of $\gamma$ is oriented). Again, not always $k_{l}+k_{r}=0$. On $\partial S$ one has only one curvature, say, $k_{l}$. In that case we set $k_{r}=0$. Finally, consider a point $\gamma(t), t \in I^{0}$ and the curves $\gamma_{1}(s)=\gamma(t-$ $s), \gamma_{2}(s)=\gamma(t+s)$. If the section angles $\alpha_{l}\left(\gamma_{1}, \gamma_{2}\right)$ and $\alpha_{r}\left(\gamma_{1}, \gamma_{2}\right)$ are defined, we define exterior angles $\beta_{l}(\gamma(t)):=\pi-\alpha_{l}\left(\gamma_{1}, \gamma_{2}\right)$ and $\beta_{r}(\gamma(t)):=$ $\pi-\beta_{r}\left(\gamma_{1}, \gamma_{2}\right)$.

Let $S$ be a 2-dimensional semi-algebraic topological manifold with boundary $\partial S$.

Notations 3.1. We assume that $S^{0}=S \backslash \partial S$ is smooth. We set $\partial S=S^{1} \cup S^{2}$ where $S^{1}$ consists of the Nash-points of $\partial S$. This includes that

i) $\partial S$ is smooth at each point $y \in S^{1}$.

ii) The tangentplane-map $p \mapsto T_{p} S$ extends continously to $S^{0} \cup S^{1}$.

By the last section $S^{2}$ can assumed to be finite.

The finite set $S^{2}$ again splits into two parts: $S^{2}=S^{21} \cup S^{22}$ where $S^{21}$ consist of those points $y$ to which the tangent plane-map extends continously, but $\partial S$ is not smooth at $y$, and $S^{22}$ consists of the remaining 
points. A semi-algebraic topological 2-manifold $S$ with boundary, for which $S^{0}$ is smooth, will be called a simple semi-algebraic surface (or briefly SSS ).

Definition 3.2. A curve $\gamma: I \rightarrow S$ is called regular, if $\gamma$ is of class $C^{1}$ in the ambient space $\mathbb{R}^{n} \supset S, \dot{\gamma}(t) \neq 0$ for all $t \in I$, and if one of the following conditions holds

i) $\gamma \subset \partial S$

ii) $\stackrel{\circ}{\gamma} \subset S^{0}, \stackrel{\circ}{\gamma}$ is of class $C^{2}$ and $\int_{\gamma}|k(t)| d t<\infty$.

Here $k$ is the inner Riemannian curvature of $\gamma$. Note that in case i) $\gamma$ can assumed to be of class $C^{\infty}$ inside $S^{1}$.

On $S^{1}$ we define the function $k$, called inner curvature, as follows: We choose a normal unit vector field $N$ along $S^{1}$, tangent to $S$ and pointing into the interior of $S$. The 1-manifold $S^{1}$ in $\mathbb{R}^{n}$ admits also a curvature vector field $\bar{k}$ along $S^{1}$. We set $k:=\langle\bar{k}, N\rangle$.

Proposition 3.3. Let $p, \in S^{1}$. Then in a neighbourhood $U$ of $p$ the local Gauß-Bonnet formula holds. Moreover, $k$ only depends on the inner metric of $S$.

More precisely, this means the following: In a neighbourhood $U$ of $p$, which is homeomorphic to a half plane and for which $\partial S \cap U \subset S^{1}$ we consider a simple closed piecewise regular curve $\gamma$ where the pieces may be parts of $S^{1}$.

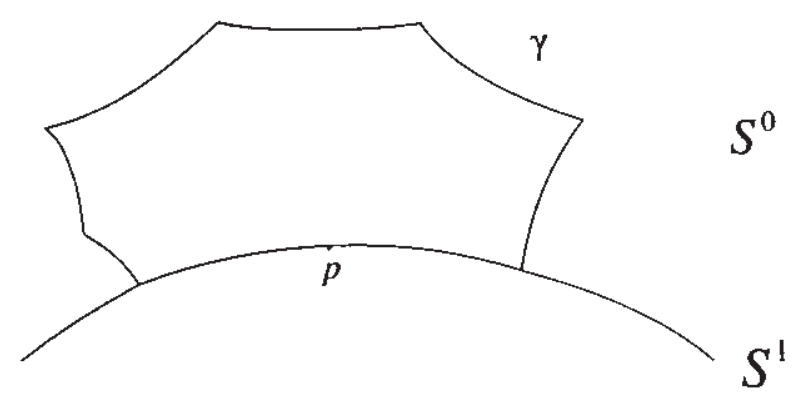

Then the inner curvature $k_{l}$ with respect to the inner part $\operatorname{Int}(\gamma)$ of the region enclosed by $\gamma$ is defined at all points of $\gamma$ and also the exterior 
angle $\beta_{i}$ at the corners $y_{i}$ of $\gamma$. Moreover, one has

$$
\int_{\operatorname{Int}(\gamma)} K(x) d x+\int_{\gamma} k_{l}(s) d s+\sum \beta_{i}=2 \pi
$$

Proof. (it might be better to say: sketch of the proof. For more details see [Ku]) We write $U$ as a Nash wing as in the previous section. Also we may assume that $\gamma$ is a triangle,

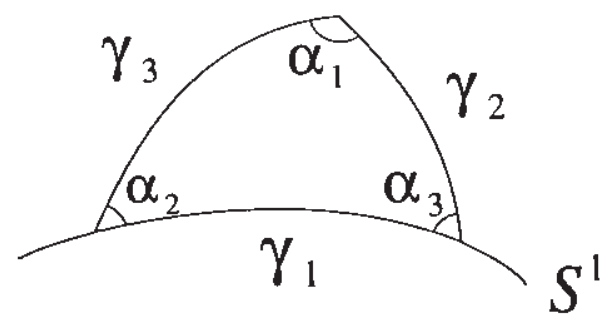

$\gamma=\gamma_{1} \circ \gamma_{2} \circ \gamma_{3}$ with $\gamma_{1} \subset S^{1}, \gamma_{2}, \gamma_{3} \subset S^{0}$ and corresponding interior angles $\alpha_{i}$ opposite to $\gamma_{i}$ for $i=1,2,3$. In the setting of Nash-wings we have

$$
\gamma_{1} \subset Y=\{(y, 0) \mid y \in Y\}
$$

We replace $Y$ by the curve $\delta=\{(y, \epsilon) \mid y \in Y\}$ for a small $y$.

For sufficiently small $\epsilon$ we get the following: A decomposition of arcs: $\gamma_{2}=\gamma_{2}^{\prime \prime} \circ \gamma_{2}^{\prime}, \gamma_{3}=\gamma_{3}^{\prime} \circ \gamma_{3}^{\prime \prime}$. And a decomposition of the triangle $\gamma=$ $\gamma_{1} \circ \gamma_{2} \circ \gamma_{3}$ into the triangle $\gamma^{\prime}=\gamma_{1}^{\prime} \circ \gamma_{2}^{\prime} \circ \gamma_{3}^{\prime}$ with $\gamma_{1}^{\prime} \subset \delta$ and with interior angles $\alpha_{3}^{\prime}, \alpha_{1}^{\prime}=\alpha_{1}, \alpha_{2}^{\prime}$ respectively, and into the quadrangle $\gamma^{\prime \prime}=\gamma_{1} \circ \gamma_{2}^{\prime \prime} \circ\left(\gamma_{1}^{\prime}\right)^{-1} \circ \gamma_{3}^{\prime \prime}$. 
We may assume that $\gamma^{\prime}$ and $\gamma^{\prime \prime}$ are again simple closed curves.

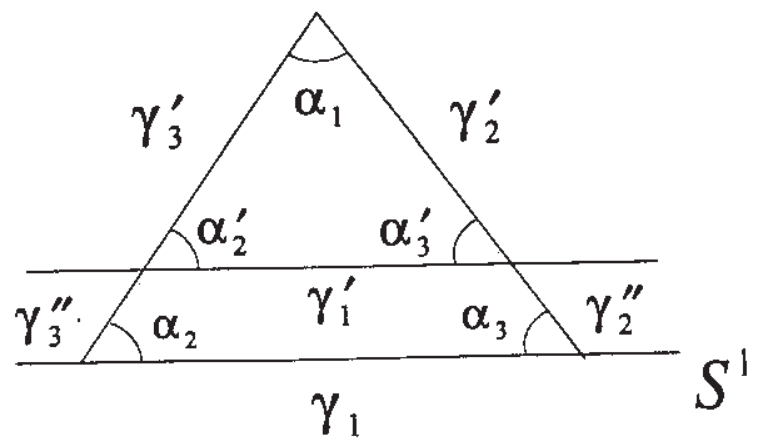

Moreover, for sufficiently small $\epsilon, \alpha_{i}^{\prime}$ is arbitrarily close to $\alpha_{i}$ and $\int_{\gamma_{i}^{\prime}} k(s) d s$ is arbitrarily close to $\int_{\gamma_{i}} k(s) d s$.

Since on the other hand $\int \operatorname{Int}\left(\gamma^{\prime \prime}\right) K d x$ gets arbitrarily small and the Gauß-Bonnet formula holds for $\gamma^{\prime}$ it also holds for $\gamma$.

Clearly, the correctness of the Gauß-Bonnet formula defines the curvature function $k$ on $S^{1}$ uniquely. Hence $k$ only depends on the interior metric of $S$.

Proposition 3.4. Let $p \in S^{21}$. Then in a neighbourhood $U$ of $p$ the local Gauß-Bonnet formula holds.

Proof. Again it is enough to consider triangles. We have the two cases as indicated in the figure below:
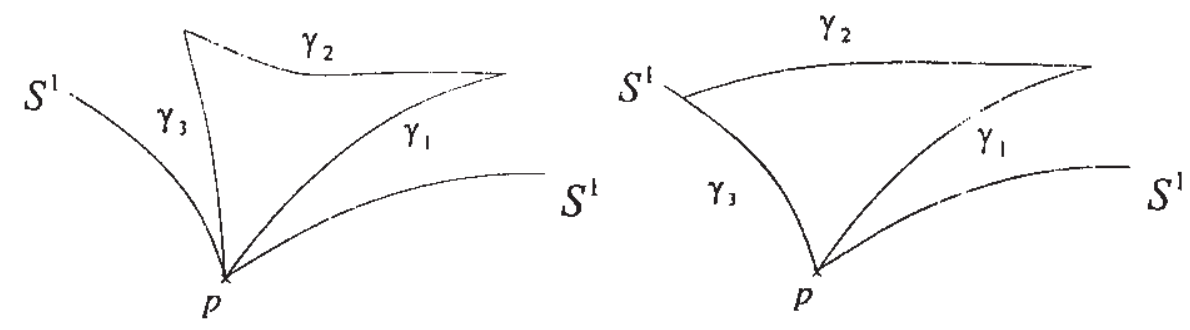

We replace $\gamma_{1}$ by a close curve $\gamma_{1}^{\prime}$ respectively, as indicated in the next 
figure,
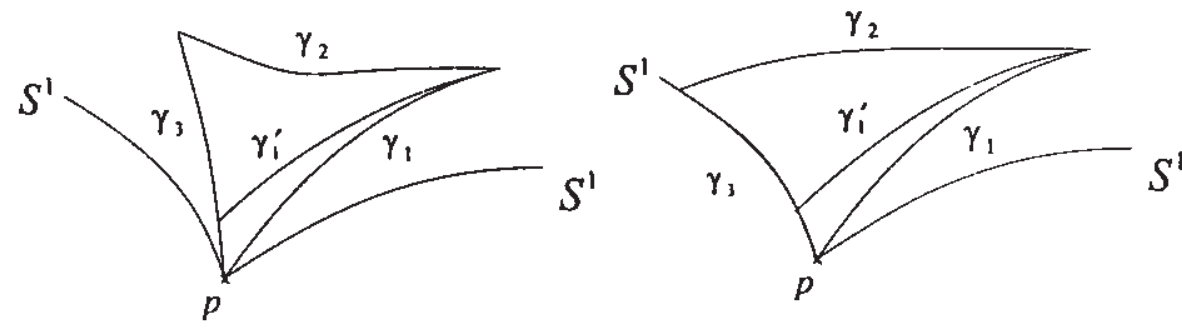

and argue similary as for proposition 3.3 , where in the second case we also use this proposition.

As a corollary of Propositions 3.3 and 3.4 we get

Proposition 3.5. With the above setting for $S$ assume that $S^{22}=\emptyset$. Let $T \subset S$ be a compact oriented 2-dimensional subvariety such that the boundary $\partial T$ is a piecewise regular curve (for instance $T=S$ ). Then

$$
\int_{T} K(x) d x+\int_{\partial T} k_{l}(s) d s+\sum_{i=1}^{n} \beta_{i}=2 \pi \chi(T)
$$

where $\beta_{i}$ is the exterior angle at the corner $p_{i}$ of $\partial T$ and $k_{l}$ the curvature with respect to Int $(T)$.

It ramains to define angles at the points $p \in S^{22}$. More generally, let $p \in S$ and let $\gamma, \delta$ be regular curves emanating from $p$ which do not intersect infinitely often in a neighbourhood of $p$. Then $\gamma$ and $\delta$ define a section, say $(\gamma, \delta)_{l}$ around $p$.

Proposition and Definition 3.6. With the above notations let $S(\rho, p)$ be the $n-1$ sphere in $\mathbf{R}^{n}$ around $p$ with sufficiently small radius $\rho$. Set $\sigma_{\rho}:=(\gamma, \delta)_{l} \cap S(\rho, p)$. Then the limit

$$
\alpha_{l}(\gamma, \delta)=\lim _{\rho \rightarrow 0} l\left(\sigma_{\rho}\right) \cdot \rho^{-1}
$$

whene $l\left(\sigma_{\rho}\right)$ is the length of $\sigma_{\rho}$ exists and defines an angle only depending on the inner metric of $S$. 
Proof. We parametize $\sigma_{\rho}$ by arc length. For $q \in \sigma_{\rho} \cap S^{0}$ we choose $Y$ and $N$ as in Lemma 2.3. Let $\bar{k}$ be the curvature in $\mathbb{R}^{n}$ of $\sigma_{\rho}$ at $q$. We get (up to a sign) $k_{l}=\langle\bar{k}, N\rangle$. Hence, by Lemma 2.3 ,

$$
k_{l}=<\bar{k}, Y>+\epsilon(\rho)=\frac{1}{\rho}+\epsilon(\varphi)
$$

where $\epsilon(\rho) \rightarrow 0$ for $\rho \rightarrow 0$. Therefore

$$
\int_{\sigma_{\rho}} d k_{l}=\frac{l\left(\sigma_{\rho}\right)}{\rho}+\epsilon(\rho) l\left(\sigma_{\rho}\right)
$$

Now consider $\tau<\rho$ and the rectangle $R$ enclosed by

$$
\eta=\gamma \mid[\tau, \rho] \sigma_{\rho}(\delta \|[\tau, \rho])^{-1} \sigma_{\tau}^{-1}
$$

As we mentioned before, the inner angles at the four corners of $R$ converge to $\pi / 2$ for $\rho \rightarrow 0$. Also, since $\gamma$ and $\delta$ have bounded curvature, we get

$$
\int_{\gamma \mid[\tau, \rho]} k_{l}(\gamma(t)) d t=\epsilon(\rho)
$$

and correspondingly for $\delta$. Hence by Proposition 3.5 we get

$$
\left|l\left(\sigma_{\rho}\right) \rho^{-1}-l\left(\sigma_{\tau}\right) \tau^{-1}\right|=\int_{R^{0}} K(x) d x+\epsilon(\rho)
$$

which shows the claim.

Corollary 3.7. In Proposition 3.5 the assumption that $S^{22}=0$ can be dropped.

Clearly, for $p \notin S^{22}$, the angle defined in 3.6 coincides with that we had before.

\section{Piecewise semi-algebraic surfaces}

We will extend our class of spaces.

Construction and notations 4.1. Assume we are given two semialgebraic 2-manifolds with boundary $S_{1}, S_{2}$ as above. Let $Y_{i}$ be a closed 
submanifold of $\partial S_{i}$ of pure dimension $1, i=1,2$ and $\varphi: Y_{1} \rightarrow Y_{2}$ an isometry. Then we get a new space $S$ by gluing $S_{1}$ and $S_{2}$ along $Y_{1}$ and $Y_{2}$ respectivly:

$$
S=S_{1} \cup S_{2} / \varphi
$$

A 2-manifold (possibly with boundary) which one gets by gluing finitely many simple semi-algebraic surfaces $S_{i}$ as above will be called a piecewise semi-algebraic surface (PSS). In fact, a semi-algebraic 2-manifold with boundary is a PSS, but the converse, in general, does not hold.

Stratification 4.2. Let $S$ be a PSS. We set $S^{0}=$ \{smooth interior points of $S$ \}. So for $p$ of $S^{0}$ a neighbourhood of $p$ is isometric to a smooth semi-algeraic 2-manifold. $S^{1}=\{$ Nash points $\}$. For $p \in S^{1}$ a neighbourhood of $p$ is isometric to a Nash-wing (if $p \in \partial S$ ) or a gluing of two Nash-wings along their boundaries (if $p \notin \partial S$ ). Again $S^{2}:=$ $S \backslash\left(S^{0} \cup S^{1}\right)$ is finite. A neighbourhood $p \in S^{2}$ comes from gluing neighbourhoods of $p_{i} \in S_{i}^{1} \cup S_{i}^{2}$ for finitely many simple semi-algebraic surfaces $S_{i}$ such that the points $p_{i} \in S_{i}$ are identified.
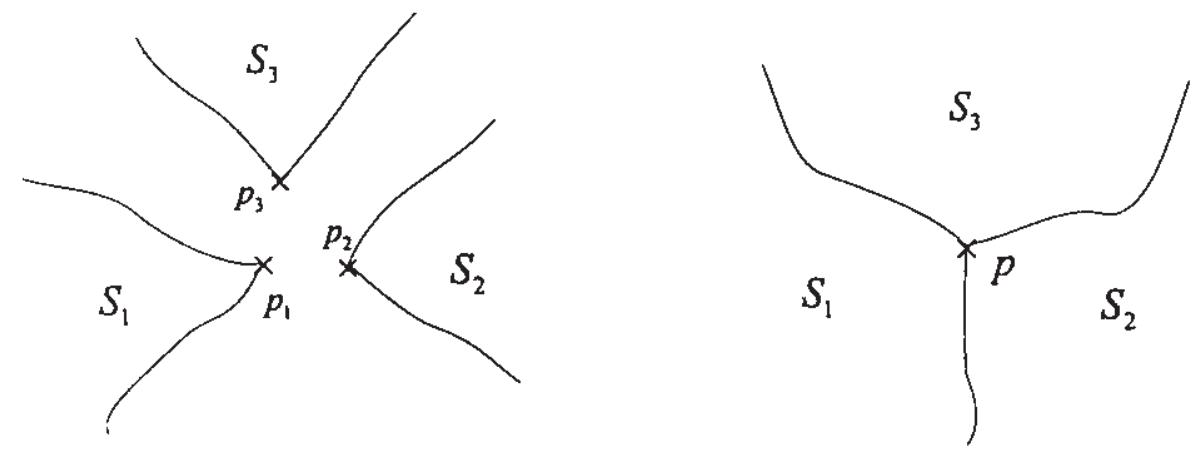

We say, that $p \in S^{21}$ if all $p_{i} \in S_{i}^{1} \cup S_{i}^{21}$ and $p \in S^{22}$ otherwise.

Let $S$ be a PSS. Then $S$ is a two-manifold with boundary. Also, if $S$ comes from gluing together simple semi-algebraic surfaces $S_{i}, i=$ $1, \ldots, r$, then one has natural isometric imbeddings

$$
\sigma_{i}: S_{i} \rightarrow S
$$

Moreover, for $p \notin S^{0}$ in a neighbourhood of $p, S^{1}$ consists of finitely many branches. We have to enlarge the class of curves we consider. 
Quasiregular curves 4.3. Let $\gamma: I \rightarrow S$ be a curve. Then there are countably many open intervals $U_{i} \subset I, i \in J$, such that $\gamma \mid U_{i} \subset S^{0}$. We set

$$
C:=I \backslash \bigcup_{i \in I} U_{i}
$$

One has $\gamma \mid C \subset S^{1} \cup S^{2}$. We make the following assumptions:

a) For all $i \in I, \gamma \mid \bar{U}_{i}$ is piecewise regular (see Definition 3.2).

b) If $\gamma(t)=p \notin S^{0}$, then there exists $\epsilon>0$ such that $\gamma \mid[t-\epsilon, t[$ (and $\gamma|| t, t+\epsilon]$ respectivly) intersects at most one branch of $S^{1}$ around $p$ (in particular, it does not meet $p$.)

Condition b) allows to define exterior angles $\beta_{l}(\gamma(t))$ and $\beta_{r}(\gamma(t))$. In fact, consider again $\gamma_{1}(s)=\gamma(t-s)$ and $\gamma_{2}(s)=\gamma(t+s)$. If $\gamma_{i}$ has infinitely many common points with a branch $\delta$ of $S^{1}$ at $\gamma(t)$, just replace $\gamma_{i}$ by $\delta$ and consider the corresponding section angle and exterior angle between $\gamma_{i}$ and $\delta$.

c) $\int_{I \backslash C}\left|k_{l}(\gamma(t))\right| d t+\sum\left|\beta_{l}(\gamma(t))\right|<\infty$.

The summation runs over all $t \in i=$ interior of $I$. A curve, which fullfills the assumptions a), b) and c) will be called quasiregular. This definition is symmetric with respect to left and right, since for almost all $t \in I$ one has $k_{l}(\gamma(t))=-k_{r}(\gamma(t))$ and $\beta_{l}(\gamma(t))=-\beta_{r}(\gamma(t))$.

Left and right curvature 4.4. Now we have to assume, that.a neighbourhood of $\gamma$ is oriented, if $\gamma$ is closed. Moreover, let $\gamma$ be quasiregular. We define left and right curvatures on $\gamma$ as regular signed measures:

$$
\int_{\gamma} d k_{l}:=\int_{\Gamma \backslash C} k_{l}(\gamma(t))+\int_{\gamma(C)} k_{l}(s) d s+\sum_{t \in \grave{I}} \beta_{l}(t)
$$

and correspondingly for the right hand side.

Curvature 4.5. Let $S$ be an oriented PSS. We have to define a regular signed measure $d K$ on $S \backslash \partial S$. So let $U \subset S \backslash \partial S$ be open. We set

$$
\int_{U} d K=\int_{U \cap S_{0}} K(x) d x+\int_{U \cap S^{1}} d k_{l}+d k_{r}+\sum_{p \in U \cap S^{2}}(2 \pi-\alpha(p))
$$


Here $0 \leq \alpha(p)$ is the total angle around $p$. (For $S S S^{\prime} s$ total angles are defined in 3.6. The extension to PSS's should be clear.) With these notations we have

Theorem 4.6. (Gauß-Bonnet-Formula) Let $S$ be a PSS and let $T \subset S$ be an oriented compact 2-dimensional subvariety such that $\partial T$ is a union of finitely many simple closed quasiregular curves. Then

$$
\int_{T \backslash \partial T} d K+\int_{\partial T} d k_{l}=2 \pi \chi(T)
$$

where $d k_{l}$ is the curvature with respect to Int $(T)$.

The proof of this is much easier then the description of all the settings above, although they are quite natural. So we leave the proof for the reader.

\section{$5 \quad$ Aleksandrovs axiom}

Let $(S, d)$ be an inner metric space such that each point $p \in S$ admits a neighbourhood $U(p)$ which is homeomorphic to an open disc in $\mathbb{R}^{2}$, that is, $S$ is a topological 2-manifold without boundary.

Geodesics and angles 5.1. A rectificable curve $\gamma:[a, b] \rightarrow S$ is called shortest geodesic, if $d(\gamma(a), \gamma(b))=l(\gamma):=$ length of $(\gamma)$. Also, $\gamma$ is called geodesic, if for all $t \in[a, b]$ there exists $\epsilon>0$ such that for all $t_{1} \leq t_{2}$ with $\left|t_{i}-t\right|<\epsilon$ one has : $\gamma \mid\left[t_{1}, t_{2}\right]$ is shortest geodesic. Next, consider two curves $\gamma_{i}:[0, e] \rightarrow S, i=1,2$, such that $\gamma_{1}(0)=\gamma_{2}(0)$. We define the upper angle $\bar{\alpha}\left(\gamma_{1}, \gamma_{2}\right)$ between $\gamma_{1}$ and $\gamma_{2}$ at $\gamma_{i}(0)$ as follows: For all $\left.t_{1}, t_{2} \in\right] 0$, e] consider the euclidean triangle with sides $g_{1}, g_{2}, c$ of length $d\left(\gamma_{1}(0), \gamma_{1}\left(t_{1}\right)\right), d\left(\gamma_{1}(0), \gamma_{2}(t)\right)$ and $d\left(\gamma_{1}(t), \gamma_{2}(t)\right)$ respectivly. Let $\alpha\left(t_{1}, t_{2}\right)$ be the angle between $g_{1}$ and $g_{2}$. Then set

$$
\bar{\alpha}\left(\gamma_{1}, \gamma_{2}\right):=\lim \sup \left\{\alpha\left(t_{1}, t_{2}\right) \mid\left(t_{1}, t_{2}\right) \rightarrow 0\right\}
$$

This $\bar{\alpha}\left(\gamma_{1}, \gamma_{2}\right)$ is called the upper angle between $\gamma_{1}$ and $\gamma_{2}$ similary, we define the angle

$$
\left.\alpha\left(\gamma_{1}, \gamma_{2}\right):=\lim \left\{\alpha\left(t_{1}, t_{2}\right)\right\}\left(t_{1}, t_{2}\right) \rightarrow 0\right\}
$$

if it exists. One has $\pi \geq \bar{\alpha}\left(\gamma_{1}, \gamma_{2}\right), \alpha\left(\gamma_{1}, \gamma_{2}\right)$. 
Triangles 5.2. Now let $U$ be an neighbourhood, homeomorphic to an open disc in $\mathbf{R}^{2}$, of some point. We consider a triangle $\triangle \subset U$ with vertices $A_{1}, A_{2}, A_{3}$ and shortest geodesics $\gamma_{k}$ connecting $A_{i}$ and $A_{j}$. We assume that the $\gamma_{k}$ fit together to a Jordan curve. Then $\triangle$ has an interior $\triangle$ and a boundary $\partial \triangle$ consisting of the three geodesic sections. Suppose that for any two points $p, q \in \partial \triangle$ and any simple arc $\gamma$ connecting $p$ and $q$ such that $\gamma$ up to the endpoints is entirely contained in $U \backslash \triangle$ one has $l(\gamma) \geq l(\delta)$ where $\delta \in \partial \Delta$ is the section which together with $\gamma$ bounds a region outside of $\AA$. Then $\Delta$ is called simple. Let $\bar{\alpha}_{i}$ be the upper angle at $A_{i}$. We define the upper defect of $\triangle$ by

$$
\bar{\alpha}_{2}+\bar{\alpha}_{3}-\pi
$$

Aleksandrovs axiom 5.3. For each $p \in S$ there exists a neighbourhood $U=U(p)$, homeomorphic to an open disc in $\mathbb{R}^{2}$, and a bound $K=K(p)$ such that for any collection $\Delta_{1}, \ldots, \Delta_{r}$ of simple triangles in $U$ with disjoint interiors one has

$$
\sum_{i=1}^{r}\left|\overline{\operatorname{def}}\left(\Delta_{i}\right)\right| \leq K .
$$

For this axiom we briefly say that $S$ has locally bounded curvature. Also $S$ is called an Aleksandrov surface. In [A-Z] it is shown, that for Aleksandrov surfaces one can develop a very strong synthetic 2dimensional differential-geometry, which shares many properties with two dimensional Riemannian geometry. However, there are also serious differences. For instance, in the synthetic setting may exist ramifying geodesics.

We will show that Aleksandrovs axiom holds for piecewise semi-algebraic spaces. We are not able to do that directly, since exterior angles are very difficult to compute in the neighbourhood of singularities. Instead of that, we will approximate piecewise semi-algebraic spaces by well behaved ones.

Of course, Aleksandrovs axiom holds for Riemannian twofolds, if they are smooth enough. This follows immediately from the Gauß-Bonnet formula and the Proposition below 
Proposition 5.4. Let $S$ be a Riemannian twofold of class $C^{2}$ and let $\gamma_{1}, \gamma_{2}$ be curves in $S$ of class $C^{1}$ emanating from the same point $p$. Then the angle between $\gamma_{1}, \gamma_{2}$ at $p$ exists and coincides with the Riemannian angle.

This is well known. See also [Sch] for a detailed proof.

We need one of the main results on Aleksandrovs surfaces

Notation 5.5. Let $X$ be a space with two metrics $d_{1}, d_{2}$. Then the Hausdorff distance $d\left(d_{1}, d_{2}\right)$ between these is defined by $d\left(d_{1}, d_{2}\right):=$ $\sup \left\{\left|d(x, y)-d^{\prime}(x, y)\right|(x, y) \in X \times X\right\}$

Theorem 5.6. Let $S$ be a topological twofold (without boundary) and let $\left(d_{n}\right) \rightarrow d$ be a Hausdorff convergent sequence of metrics on $S$. Assume that all $d_{n}, d$ are inner and compatible with the topology of $S$. If $\left(S, d_{n}\right)$ has uniformly locally bounded curvature, then $(S, d)$ is an Aleksandrov surface.

Proof. [A-Z, Chap. IV, Th. 15 in connection with IIl, Th. 11 f.f.]

\section{Piecewise semi-algebraic surfaces are Aleksandrov surfaces}

We are going to show the statement of the headline.

Theorem 6.1. Let $S$ be a piecewise semi-algebraic surface without boundary. Then Aleksandrovs axiom holds for $S$.

The proof is done in two steps: First, by cutting and pasting we replace the metric $d$ on $S$ by a metric $d^{\prime}$ which is close to $d$ and for which the axiom holds, and then we use approximation according to Theorem 5.6.

1. Recall that $S$ is built up from simple semi-algebraic surfaces $S_{i}$ by pasting pieces of the boundaries. We replace the metric on each $S_{i}$. Let $p \in S_{i}^{2}$. Let $B(p, \epsilon)$ be a small ball of radius $\epsilon$ around $p$. By Theorem 2.3, $B(p, \epsilon) \cap S_{i}$ is homeomorphic to a triangle $\Delta$ with sides $a, b, c$ where $c$ is opposite to $p$. Taking the original length's of the sides, we may have $l(c)>l(a)+l(b)$. We consider a spiral $\sigma(t), t \in[0,1]$ in $\mathbf{R}^{2}$ around the origin, such that $\|\sigma(o)\|=l(a),\|\sigma(1)\|=l(b)$ and $l(\sigma)=l(c)$. We 
divide $\sigma$ into $k$ sections $T_{j}$ for which the angle at the origin is smaller than $\pi$. Then we cut off $B(p, \epsilon) \cap S$ from $S$ and replace it by pasting $T_{j}$ along $\sigma$ and $S(p, \epsilon) \cap S$ and the common edges of the $T_{j}$ :
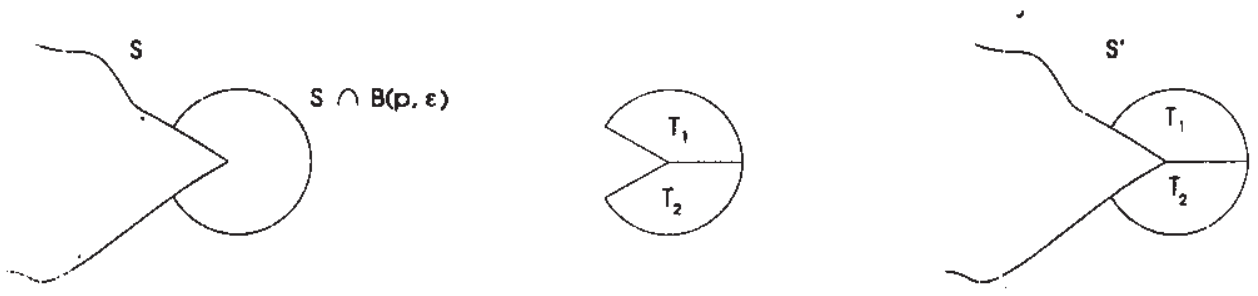

Here $S(p, \epsilon)$ is the sphere of radius $\epsilon$ around $p$. Note that the number $k$ of sections $T_{j}$ and the total curvature of $\sigma$ in $\mathbb{R}^{2}$ can be bounded independently of $\epsilon$. After identifying the new surface $S^{\prime}$ with $S$, we get a new metric $d^{\prime}$ on $S$ which is close to $d$ for small $\epsilon$. By construction it is easly achieved that $d\left|\partial S=d^{\prime}\right| \partial S$. We do this replacement for each $p \in S_{i}^{2}$. Let

$$
R_{i}:=S_{i} \backslash \cup_{p \in S_{i}^{2}} B(p, \epsilon) .
$$

Then at $R_{i} \cap S_{i}^{1}$ we have finitely many Nash-wings. Here we replace a tubular $\epsilon$-neighbourhood $T_{\epsilon}$ of $S_{i}^{1}$ in such a Nash-wing by an euclidean quadrangle of corresponding side lengths.

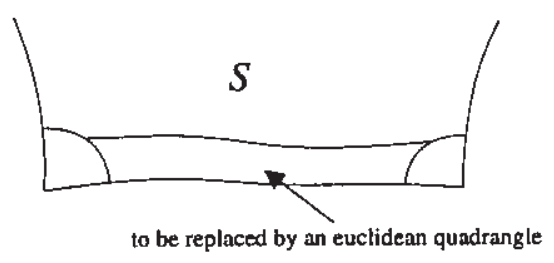

If $T_{\epsilon}$ is unbounded at both sides (half bounded), we replace by a euclidean parallel strip (half parallel strip). For half parallel strips one has only to adapt the bounded side length. Again, it is easy to identify the new surface $S^{\prime}$ with $S$ in a way such that the inner 'metric $d^{\prime}$ coming 
from $S^{\prime}$ is close to $d$ for small $\epsilon$, and $d\left|\partial S=d^{\prime}\right| \partial S$.

Finally we point out, that we have a description of the curves $S(p, \epsilon) \cap S_{i}$ and $\partial T_{\epsilon} \cap S_{i}$ the complexity of which only depends on the complexity of a description for $S_{i}$ but not on $\epsilon$. Hence the total absolute curvature of these curves is uniformly bounded, independently of $\epsilon$ (see Proposition 2.2). We do this for all $S_{i}$ and patch them together as before. Thus we get an inner metric $d_{\epsilon}$ on $S$ so that $\left(S, d_{\epsilon}\right)$ again is a PSS and $d_{\epsilon}$ tends to $d$ for $\epsilon \rightarrow 0$.

2. Now Aleksandrovs axiom holds for $\left(S, d_{\epsilon}\right)$. This follows from $\mid \mathrm{A}-\mathrm{Z}$, Chap. IX, 3]. In our situation this can also be seen directly. In fact, a geodesic arc in $\left(S, d_{\epsilon}\right)$ is easily seen to be quasiregular (for a detailed proof see [Sch]). Moreover, each building block of $\left(S, d_{\epsilon}\right)$ is completely contained in a $C^{\infty}$-Riemannian twofold. Therefore, by Proposition 5.4, upper angles between geodesics coincide with the Riemannian angles. Hence, by the Gauß-Bonnet formula, for a simple triangle $\triangle$ we have $\operatorname{def}(\Delta)=\int_{\Delta} d K$

Now, by the definition of $d K$ (see 4.5) and the first part of the proof we even get: There is a bound $\rho$, independent of $\epsilon$, such that for $\left(S, d_{\epsilon}\right)$ one has $\int_{S} d K \leq \rho$. In particular, the family $\left\{\left(S, d_{\epsilon}\right) \mid \epsilon>0, \epsilon\right.$ sufficiently small \} has uniformly locally bounded curvature. Since $d_{\epsilon} \rightarrow d$ for $\epsilon \rightarrow 0$, by Theorem 5.6 it follows that $(S, d)$ is an Alekandrov surface too.

\section{Angles, directions and curvature of curves}

We want to apply the theory of Aleksandrov surface to piecewise semialgebraic surfaces $S$. To this end we have to review some notations and results (see [A-Z] for a general reference).

Angles and directions 7.1. Let $S$ be an Aleksandrov surface. Let $p \in S$ and $\gamma$ a curve emanating from $p$. We say that $\gamma$ has a direction at $p$ if the angle $\alpha$ between $\gamma$ and $\gamma$ at $p$ exists. Then necessarily $\alpha=0$. If $\gamma$ is geodesic, it has obviously a direction at $p$. If there is a second curve $\delta(s)$ emanating from $p$ and having a direction at $p$, then also the angle $\beta=: \alpha(\gamma, \delta)$ between $\gamma$ and $\delta$ exists [A-Z, Chap. VI, Th. 1]. If, moreover, $\gamma$ and $\delta$ have infinitely many common points in any neighbourhood of 
$p$, then $\beta=0$. If $\beta=\pi$, possibly $\beta$ has no geometrical meaning. For instance, this occurs if $S$ is piecewise semi-algebraic, $p \in S^{2}$ and the total angle at $p$ exceeds $2 \pi$. Therefore one defines sector angles as follows. Assume $\gamma$ and $\delta$ have no common points $\neq p$ in some neighbourhood of $p$. Then $\gamma$ and $\delta$ define two sectors $(\gamma, \delta)_{l}$ and $(\gamma, \delta)_{r}$.

Consider in such a sector, say $(\gamma, \delta)_{l}$ a sequence $\gamma=\gamma_{0}, \gamma_{1}, \gamma_{2}, \ldots, \gamma_{r}=\delta$ of simple continuous curves emanating from $p$, having directions at $p$ and being labeled in their natural order. We set

$$
\alpha\left(\gamma_{0}, \ldots, \gamma_{r}\right)=\sum_{i=0}^{r-1} \alpha\left(\gamma_{i}, \gamma_{i+1}\right)
$$

and

$$
\alpha(\gamma, \delta)_{l}=\sup \left\{\alpha\left(\gamma_{0}, \ldots, \gamma_{r}\right)\right\}
$$

Moreover, we set

$$
\alpha(p)=\alpha(\gamma, \delta)_{l}+\alpha(\gamma, \delta)_{r} \text { (total angle at } p \text { ) }
$$

If any two geodesics emanating from $p$ have common points arbitrarily close to $p$ we set $\alpha(p)=0$. Let $\gamma, \delta$ define sectors as above, and let $\alpha(\gamma, \delta)$ the angle between $\gamma$ and $\delta$ at $p$. One has

a) $\alpha(\gamma, \delta)=\min \left\{\alpha(\gamma, \delta)_{l}, \alpha(\gamma, \delta)_{r}, \pi\right\}$

b) If $\eta \in(\gamma, \delta)_{l}$ has a direction at $p$, then $\alpha(\gamma, \delta)_{l}=\alpha(\gamma, \eta)_{l}+\alpha(\eta, \delta)_{l}$

c) If $(\gamma, \delta)_{l}$ is convex, then $\alpha(\gamma, \delta)=\min \left\{\alpha(\gamma, \delta)_{l}, \pi\right\}$

From b) it follows that the total angle $\alpha(p)$ is well defined. For more details see [A-Z, Chap. IV, 4].

Curvature of curves 7.2. Let $\gamma:[a, b] \rightarrow S$ be a curve with directions at the end points $p=\gamma(a)$ and $q=\gamma(b)$. We also assume that $\alpha(p) \neq$ $0, \alpha(q) \neq 0$. If $\gamma$ consists piecewise of shortest geodesics, $\gamma$ is called a polygone. In a suitable small neighbourhood $\gamma$ has a left and a right hand side. In particular, if $\gamma$ is a polygone and $p_{i} \neq p, q$ a vertex, we have left and right exterior angles $\beta_{l}\left(p_{i}\right)$ and $\beta_{r}\left(p_{i}\right)$ defined by $\gamma$ at $p_{i}$. We set

$$
\bar{k}_{l}(\gamma)=\sum_{p_{i}} \beta_{l}\left(p_{i}\right), \quad \bar{k}_{r}(\gamma)=\sum_{p_{i}} \beta_{r}\left(p_{i}\right)
$$


where $p_{i}$ runs over the vertices $\neq p, q$ of $\gamma$.

Now assume, that $\gamma$ is not necessarily polygonal, and let $\gamma_{i}$ be a sequence of polygones with endpoints $p, q$ which converges to $\gamma$ from the left hand side (so that $\gamma_{i} \cap \gamma$ consists of vertices only). Then, locally at $p$ and $q$ we have regions enclosed by $\gamma_{i}$ and $\gamma$ and corresponding sectorangles $\sigma_{i}$ and $r_{i}$. The sequence $\left(\bar{k}_{l}\left(\gamma_{i}\right)+\sigma_{i}+\tau_{i}\right)$ tends to a well defined value $\mid \mathrm{A}-\mathrm{Z}$, Chap. IV, Th. 2] which is called left curvature ([A-Z] uses the word "left rotation") and denoted by $k_{l}(\gamma)$. If $\gamma$ is polygone, then $\bar{k}_{l}(\gamma)$ and $k_{l}(\gamma)$ in general do not coincide.

Next, let $\gamma: I \rightarrow S$ be a curve. We say that $\gamma$ has bounded curvature (in [A-Z] one says more precisely "bounded variation of curvature") if the following conditions hold:

a) At all $p \in \gamma$ the total angle $\alpha(p) \neq 0$.

b) For all $p \in \dot{\gamma}, \gamma$ has left and right directions and hence left and right exterior angles $\beta_{l}(p)$ and $\beta_{r}(p)$.

c) There is a bound $\rho$ such that for all ordered sequences of points $p_{1}, \ldots, p_{r} \in \stackrel{\circ}{\gamma}$ one has

$$
\sum_{i=1}^{r+1}\left(\left|k_{l}\left(\gamma_{i}\right)\right|+\left|k_{r}\left(\gamma_{i}\right)\right|\right)+\sum_{i=1}^{r}\left(\left|\beta_{l}\left(p_{i}\right)\right|+\left|\beta_{r}\left(p_{i}\right)\right|\right)<\rho .
$$

Here $\gamma_{1}, \ldots, \gamma_{r+1}$ are the sections of $\gamma$ defined by the $p_{i}$.

If $\gamma$ has bounded curvature, one has natural regular signed measures $d k_{l}$ and $d k_{r}$ on $\gamma$. [A-Z, Chap. LX, Th. 1]

Examples 7.3. Assume that $\alpha(p) \neq 0$ for $p \in \gamma$.

a) Let $\gamma$ be a geodesic in $S$. Then $\gamma$ has bounded curvature. However, it is not true in general, that $d k_{l}=d k_{r}=0$. One only has $d k_{l} \leq$ $0, d k_{r} \leq 0$.

b) Let $S$ be piecewise semi-algebraic, and let $\gamma$ be a (quasi)regular curve in $S$. Then $\gamma$ has bounded curvature and the set functions $d k_{r}$ and $d k_{l}$ coincide with those we defined in 4.4. We will apply this for the special case that $\gamma$ is a semi-algebraic path. 
Proposition 7.4. Let $\gamma:[a, b] \rightarrow S$ be a curve of bounded curvature. Then $\gamma$ has directions at the endpoints.

Proof. [A-Z, Chap. IX, Th. 2].

Gauss-Bonnet formula 7.5. There is a canonical regular signed measure $d K$ on $S[\mathrm{~A}-\mathrm{Z}$, Chap: V]. Let $T \subset S$ be a compact oriented submanifold of dimension 2 with boundary $\partial S$. So $\partial S$ consists of finitely many closed curves $\gamma_{1}, \ldots, \gamma_{r}$. Then

$$
\sum_{T^{\circ}} d K+\sum_{i=1}^{\tau} k_{l}\left(\gamma_{i}\right)=2 \pi \chi(T)
$$

Where the left hand side is the inner side of $T$.

Proof. [A-Z, Chap. VI, Th. 5].

\section{Piecewise semi-algebraic surfaces revisted}

Now let $S$ be a piecewise semi-algebraic surface. Since $S$ is also an Aleksandrov surface, we have many things doubly defined: sector angles, curvature on $S$ and left and right curvatures of curves. We have also two Gauß-Bonet formulas. These will help us to identify these quantities. Let us provide the notations we had for piecewise semi-algebraic surfaces with tildes. First we show

Lemma 8.1. Let $p \in S^{1}, \delta$ a branch of $S^{1}$ at $p$ and $\gamma$ a geodesic emanating from $p$ which intersects $\delta$ in any neighbourhood of $p$. Then $\gamma$ is of class $C^{1}$ at $p$ and $\tilde{\alpha}(\gamma, \delta)=0$.

Proof. The projection of $S$ into the tangent space $T_{p} S$ defines a semialgebraic $C^{1}$ chard $p r: S \rightarrow \mathbf{R}^{2}$ in a neighbourhood of $p$ and the origin respectively. It is enough to show that the claim holds for $\operatorname{pr}(\gamma)$ and $\operatorname{pr}(\delta)$ at 0 . If the claim does not hold we have a linear ray $\eta$, starting at 
0 , which includes with $\operatorname{pr}(\delta)$ a positive angle $\beta$, so that $\gamma$ also interserts $\eta$ in any neighbourhood of 0 .

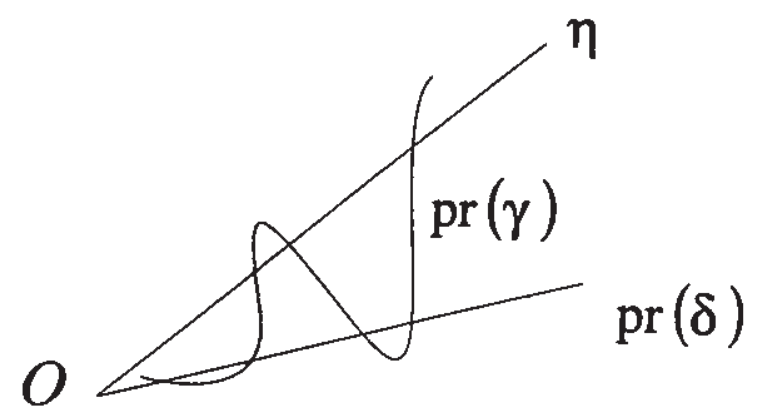

Then in any neighbourhood of 0 there are pieces of $\operatorname{pr}(\gamma) \subset \operatorname{pr}\left(S^{0}\right)$ the total curvature of which exceeds a certain value. Since the map $q \stackrel{\leftrightarrow}{\mapsto} T_{q} S$ (in some ambient space) extends continuously to $S^{1}$, the variation of the curvature on $\gamma$ converges to that on $p r(\gamma)$. On the other hand, $\gamma$ being geodesic has no curvature on $S^{0}$. Contradiction. Similary it follows, that the right derivation on $\gamma$ is continuous.

Lemma 8.2. Under the assumptions of Lemma 8.1 assume we have a second geodesic $\gamma_{1}$ which intersects $\delta$ in any neighbourhood of $p$. Then $\alpha\left(\gamma, \gamma_{1}\right)=\tilde{\alpha}\left(\gamma, \gamma_{1}\right)=0$.

Proof. Clearly $\tilde{\alpha}\left(\gamma, \gamma_{1}\right)=0$. Also $\alpha\left(\gamma, \gamma_{1}\right)=0$ if $\gamma$ and $\gamma_{1}$ intersect in any neighbourhood of $p$. Assume first, that in any neighbourhood of $p$ there exist $q \in \gamma \cap S^{0}, q_{1} \in \gamma_{1} \cap S^{0}$ and a $C^{2}-$ arc $\eta$ in $S^{0}$ from $p$ to $q$ such that we get a triangle $\Delta$ with vertices $p, q, q_{1}$ enclosed by a simple closed curve consisting of a section of $\gamma, \gamma_{1}$ and $\eta$. For such a triangle the defect tends to zero (for $d K$ and $d \tilde{K}$ ) and the angles at $q, q_{1}$ coincide in both settings. Hence both Gauß-Bonnet formulas yield, that also $\alpha\left(\gamma, \gamma_{1}\right)=\tilde{\alpha}\left(\gamma, \gamma_{1}\right)$. 
In the remaining case, $\gamma$ and $\gamma_{1}$ may have common tangents with $\delta$, arbitrarily close to $p$. Then we choose a shortest geodesic $\gamma_{2}$ connecting a point "between" $\gamma$ and $\gamma_{1}$ with $p$.

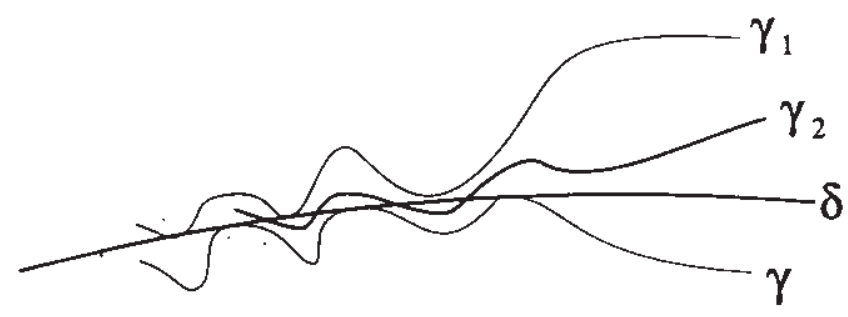

In the situation of the figure by what we have shown we get $\alpha\left(\gamma, \gamma_{2}\right)=$. $0=\alpha\left(\gamma_{2}, \gamma_{1}\right)=\alpha\left(\gamma, \gamma_{1}\right)$. Still it might happen that $\gamma_{2}$ remains on the same side of $\delta$ than $\gamma_{1}$ but then one can achieve that $\gamma_{2}$ has a common point with $\gamma$, arbitrarily close to $p$. It follows $\alpha\left(\gamma, \gamma_{1}\right)<\epsilon$ and $\alpha\left(\gamma_{1}, \gamma_{2}\right)<$ $\epsilon$.

Lemma 8.3. Let $p$ and $\delta$ be as in the previous Lemmata. Then $\delta$ has a direction at $p$.

Proof. Assume that the claim does not hold. Then there are geodesics $\gamma, \gamma_{1}$ emanating from $p$ such that $\alpha\left(\gamma, \gamma_{1}\right)>0$ and $\delta$ intersects $\gamma$ and $\gamma_{1}$ in any neighbourhood of $p$. Contradiction to Lemma 8.2.

Lemma 8.4. Let $\gamma$ be a regular curve in $S$ emanating from $p \in S$ with $\alpha(p) \neq 0$ such that, apart from $p, \gamma$ is contained in $S^{0}$. Then $\gamma$ has a direction at $p$. Moreover, $d k=d \vec{k}$ on $\gamma$.

Proof. First we show that for $\gamma$ in $S^{0}$ we have $d k=d \tilde{k}$. For this let us first assume that $\gamma$ is geodesic, $\gamma \subset S^{0}$. Then $d k_{l} \leq 0$ and $d k_{r} \leq 0$. Of course we have $d K=d \tilde{K}$ on $S^{0}$. Hence $0 \geq \int_{\gamma}\left(d k_{l}+d k_{r}\right)=\int_{\gamma} d K=$ $\int_{\gamma} d \tilde{K}=0$. The first equality is by [A-Z, Chap. VI, Th. 6]. It follows 
$d k_{l}=d k_{r}=0$. Next let $\gamma \subset S^{0}$ be regular. For small curves $\gamma$ we may consider a simple triangle in $S^{0}$ with sides $\gamma, \delta, \eta$ where $\delta$ and $\eta$ are geodesics. We know, by Proposition 5.4, that for the angles we have $\alpha_{i}=\tilde{\alpha}_{i}, i=1,2,3$. Comparing the Gauf-Bonnet formulas we get $\int_{\gamma} d k=\int_{\gamma} d \bar{k}$

Now we allow that the startpoint $p$ of $\gamma$ is possibly not in $S^{0}$. However, by Proposition 7.4, $\gamma$ has a direction at $p$.

Proposition 8.5. Let $p \in S^{0} \cup S^{1}$ and let $\gamma_{1}, \gamma_{2}$ be regular curves emanating from $p$. Then $\alpha\left(\gamma_{1}, \gamma_{2}\right)=\tilde{\alpha}\left(\gamma_{1}, \gamma_{2}\right)$ and correspondingly for section angles at $p$. In particular, for the total angle at $p$ one has $\tilde{\alpha}(p)=$ $\alpha(p)=2 \pi$.

Proof. We need only to show the first claim. Since $\alpha\left(\gamma_{1}, \gamma_{2}\right)$ exists, we can compute it by considering sequences $\left(\gamma_{1}\left(t_{n}\right)\right),\left(\gamma_{2}\left(s_{n}\right)\right), t_{n} \rightarrow 0, s_{n} \rightarrow$ 0 for $\gamma_{i}:\left[0, \epsilon\left[\rightarrow S\right.\right.$, such that $d\left(p, \gamma_{1}\left(t_{n}\right)\right)=d\left(p, \gamma_{2}\left(s_{n}\right)\right) \neq 0$. In this situation the claim follows easily from the continuity of the Riemann metric at $p$.

Corollary 8.6. (Reflection law) Let $p \in \cdot S^{1}, \delta_{1}, \delta_{2}$ the two branchs of $S^{1}$ at $p$ and let $\gamma$ be a geodesic passing through $p$ with branchs $\gamma_{1}, \gamma_{2}$ emanating from $p$. Then $\tilde{\alpha}\left(\gamma_{1}, \delta_{1}\right)=\tilde{\alpha}\left(\gamma_{2}, \delta_{2}\right)$.

Proof. One has $\alpha\left(\gamma_{1}, \delta_{1}\right)+\alpha\left(\gamma_{2}, \delta_{1}\right) \geq \pi$ and $\alpha\left(\gamma_{1}, \delta_{2}\right)+\alpha\left(\gamma_{2}, \delta_{2}\right) \geq \pi$. [A-Z, Chap. VI, Th. 6]. On the other hand $\alpha\left(\gamma_{1}, \delta_{1}\right)+\alpha\left(\gamma_{2}, \delta_{1}\right)+$ $\alpha\left(\gamma_{1}, \delta_{2}\right)+\alpha\left(\gamma_{2}, \delta_{2}\right)=2 \pi$.

Corollary 8.7. $d K=d \tilde{K}$.

Proof. This is clear on $S^{0}$. Next let $\gamma$ be a small arc in $S^{1}$. In a small neighbourhood $U$ of $\gamma$ we choose a piecewise regular closed curve $\eta$ which 
intersects $S^{1}$ at the endpoints of $\gamma$.

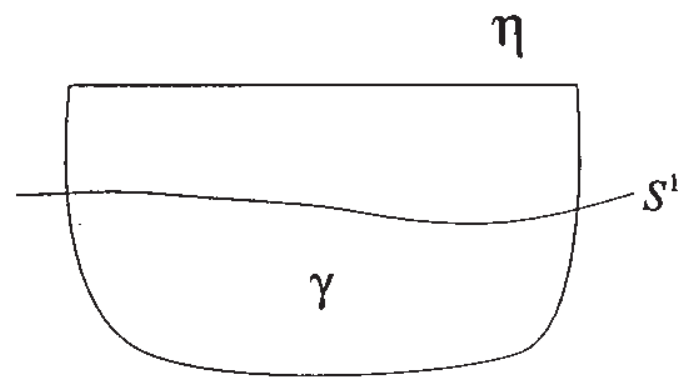

By Proposition 5.4, Lemma 8.4 and Proposition 8.5 we know that $d k=$ $d \vec{k}$ on $\eta$, hence

$$
\int_{\operatorname{Int}(\eta)} d K=\int_{\operatorname{Int}(\eta)} d \tilde{K}
$$

Since $d K$ is regular, for small $U$ the left hand side tends to

$$
\int_{\gamma} d K=\int_{\gamma} d k_{r}+\int_{\gamma} d k_{l}=\int_{\gamma} d \bar{k}_{l}+\int_{\gamma} d \bar{k}_{r} .
$$

The left equality holds by [A-Z, Chap. VI, Th. 3] and the right one by Theorem 4.6.

Similary, a point $p \in S^{2}$ can be enclosed by a small simple piecewise regular curve. Then, using the same argument, we get

$$
K(p)=\tilde{\alpha}(p)-2 \pi=\tilde{K}(p)
$$

From this proof we see furthermore

\section{Corollary 8.8 .}

a) Let $\gamma$ be a regular curve in $S$ with $\alpha(p) \neq 0$ for all $p \in \gamma$. Then $d k=d \bar{k}$ on $\gamma$.

b) For $p \in S$ with $\alpha(p) \neq 0$ and any two regular curves $\gamma_{1}, \gamma_{2}$ emanating from $p$ one has $\alpha_{l}\left(\gamma_{1}, \gamma_{2}\right)=\tilde{\alpha}_{l}\left(\gamma_{1}, \gamma_{2}\right)$ and $\alpha_{r}\left(\gamma_{1}, \gamma_{2}\right)=$ $\tilde{\alpha}_{r}\left(\gamma_{1}, \gamma_{2}\right)$. 
c) For all $p \in S, u(p)=\tilde{\alpha}(p)$.

Proof. For part b) only the case where $p \in S^{2}$ has not yet been considered. This can again been done by comparing both Gauf-Bonnet formulas. Part c) follows from the fact that also $K(p)=\alpha(p)-2 \pi$.

Part a) extends to quasiregular curves. We will not enter into this since quasiregularity is just an auxiliary notation. For the remaining part of this paper let $S \subset \mathbb{R}^{n}$ be a semi-algebraic twofold without boundary. This is the situation we are originally interested in. However, our intrisic approach shows that many properties of $S$ do not depend on the special presentation of $S$. In particular, we get some insight into the behavior of geodesics.

Proposition 8.9. Let $S \subset \mathbf{R}^{n}$ be semi-algebraic and let $\gamma$ be a curve with bounded curvature in $S$. Then at all points $p$ on $\gamma$ the curve $\gamma$ in $\mathbb{R}^{n}$ has left and right derivatives respectively and these are left (resp.) right continuous.

Proof. We may assume that $p \in S$ with $\alpha(p) \neq 0$ is the initial point of $\gamma$. Chose a semi-algebraic chart $\varphi: U \rightarrow V \subset \mathbf{R}^{2}$ where $U$ is a neighbourhood of $p, V$ is an open disc with center 0 and $\varphi(p)=0$. We set $V=\left\{t y \mid t \in\left[0,1[, y \in C\}\right.\right.$ where $C \subset \mathbf{R}^{2}$ is the unit circle. Then we can choose $\varphi$ in such a way that the following conditions hold:

i) For fixed $y \in C$ the curve $c_{y}:\left[0,1\left[\rightarrow U ; t \mapsto \varphi^{-1}(t y)\right.\right.$ is of class $C^{1}$.

ii) The map $\left[0,1\left[\times C \rightarrow \mathbf{R}^{n} ;(t, y) \mapsto c_{y}(t)\right.\right.$ is continuous.

Moreover, for $\epsilon>0$ the chart can be chosen in such a way, that uniformly

$\left|\int_{I} k(t) d t\right|<\epsilon$ where $I \subset c_{y}, y \in C$ and $k(t)$ is the curvature on $c_{y}$.

This can be seen by using resulution of singularities for the Zariskiclosure of $S$. For the last statement see also the argument in Lemma 2.3. Let $\sum$ be the bundle of all curves $c_{y}(t)$ for $y \in C$. Since $\alpha(p)>0, \gamma$ can only finitely often turn around $p$. [A-Z, Chap. IX, Th. 2]. Therefore, if $\gamma$ has no right derivative at $p$, there are $\sigma_{1}$ and $\sigma_{2} \in \sum$ having different 
right derivatives in $\mathbb{R}^{n}$, such that $\gamma$ runs infinitely often back and forth between $\sigma_{1}$ and $\sigma_{2}$.

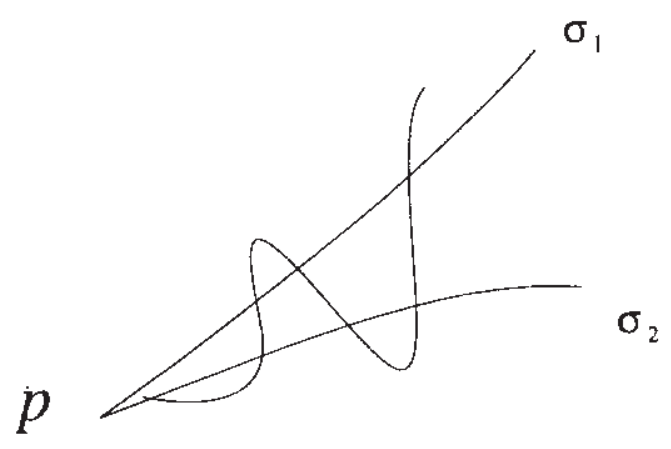

It follows, that $\gamma$ shares the direction at $p$ with $\sigma_{1}$ and $\sigma_{2}$. Contradiction.

Similary, for any $\epsilon>0$ there exists $\rho>0$ such that in $B(\rho, p)$ one has: $\gamma$ is contained in a section $\left(\sigma_{1}, \sigma_{2}\right)_{l}, \sigma_{1}, \sigma_{2} \in \sum$, such that $\alpha_{l}\left(\sigma_{1}, \sigma_{2}\right)<\epsilon$. Therefore, if $q \in \gamma \cap B(\rho, p)$ and $\sigma \in \sum$ with $q \in \sigma$, then, at $p, \alpha_{l}(\sigma, \gamma)<$ $\epsilon$. Also, for $q$ close to $p$, the right derivative of $\gamma$ at $q$ becomes close to the left derivative. If the latter is the same as the left derivative of $\sigma$ at $q$, we are done. Otherwise there is a first point $r$ (possibly $p$ ) where $\gamma$ and $\sigma$ meet again.

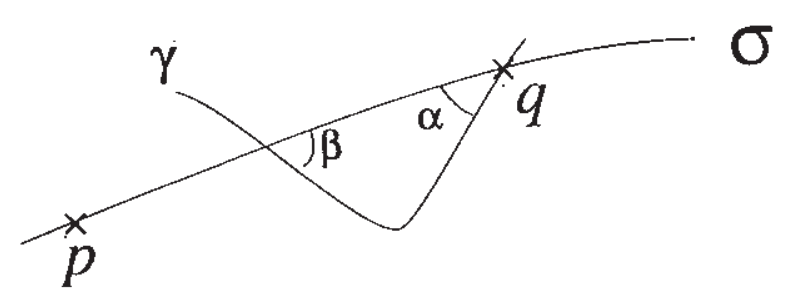

By the Gauf-Bonnet formula, the angles $\alpha$ and $\beta$, as in the figure, get small if $q$ is close to $p$. This shows, that the right derivatives of $\gamma$ are continuous. 
Corollary 8.10. Let $S \subset \mathbf{R}^{n}$ be semi-algebraic, $p \in S$ with $\alpha(p) \neq 0$ and let $\gamma$ be a curve, emanating from $p$, such that $\gamma$ is geodesic. Then $\gamma$ has continuous right derivatives.

Proof. It is known, that geodesics in Aleksandrov surfaces have bounded curvature. In our situation, one can easily compute it:

$$
\int_{\gamma} d k_{l}=\int_{\gamma \cap S^{1}} d k_{l}+\sum_{p \in \gamma^{0}} \beta_{l}(p)
$$

where $\beta_{l}(p)$ is the left exterior angle at $p$. Note that $\beta_{l}(p) \leq 0$ for all $p \in \gamma^{0}$ and for almost all of them one has equality.

It is not hard to show the preceding result directly, if $p \notin S^{22}$, but for $p \in S^{22}$ we were not able to do that. Next we will get rid of the assumption that $\alpha(p) \neq 0$.

Proposition 8.11. For $p \in S$ the following conditions are equivalent:

a) The total angle $\alpha(p)=0$

b) All semi-algebraic arcs emanating from $p$ have the same right derivative in $\mathbf{R}^{n}$.

Proof. We have $\alpha(p)=\tilde{\alpha}(p)=\lim _{\rho \rightarrow 0} l\left(\sigma_{\rho}\right) \rho^{-1}$ (see Definition 3.6).

Of course, this result is expected, but we were not able to get it by direct computation of angles in Aleksandrov surfaces. If $\alpha(p)=0$, automatically all curves $\gamma$ emanating from $p$ have directions and also right derivatives in $\mathbf{R}^{n}$. The angle between two of them is zero. So quite generally it holds that $\tilde{\alpha}_{l}\left(\gamma_{1}, \gamma_{2}\right)=\alpha\left(\gamma_{1}, \gamma_{2}\right)$ if both angles are defined. However, there may exist curves $\gamma$ emanating from $p$ such that $\stackrel{\circ}{\gamma}$ is geodesic but the right derivative is not continuous. Then $\gamma$ turns infinitely often around $p$ with respect to each semi-algebraic chart.

Natural stratification 8.12. Let $S$ be semi-algebraic. Then $S$ admits a natural semi-algebraic stratification which only depends on the inner metric of $S$ : We set $T^{n}=$ set of points which admit a Riemannian 
neighbourhood, where the fundamental form is of class $C^{n}$. Then $S^{0} \mathrm{C}$ $T^{\infty}$. Moreover, $T^{0} \backslash S^{0}$ consists of those points $p$ of $S^{1}$, where $d k_{l}=$ $-d k_{r}$ in a neighbourhood of $p$ and possibly certain points $p \in S^{2}$ where necessarily $\alpha(p)=2 \pi$. Hence $T^{0} \backslash S^{0}$ is semi-algebraic. From this one can show that $T_{0} \backslash T^{i}$ is a semi-algebraic subset of $S^{1} \cup S^{2}$ for $i<\infty$. Also $R^{1}:=\left(S^{1} \cup S^{2}\right) \backslash T^{0}$ is semi-algebraic. It consists of those points $p$, which admit neighbourhoods $U$ such that $\operatorname{vol}(U)$ is arbitrarily small but $\left|\int_{U} d K\right|$ is bigger than some constant. In $R^{1}$ in turn we have a finite set of very singular points: Ramification points and points $p$, for which $\alpha(p) \neq 2 \pi$. To the latter belong the isolated points of $R^{1}$.

\section{References}

[A-Z] Aleksandrov, A.D., Zalgaller, V.A., Intrinsic Geometry of Surfaces, AMS Translations of Monographs 15, 1967.

[B-C-R] Bochnak, J., Coste, M., Roy, M.F., Géométrie Algébrique Réelle. Ergebnisse der Mathematik, Springer-Verlag, Berlin, Heidelberg, New York 1986.

[Eu] Eulering, G., Integrale Krümmungskonstanten algebraischer Untermannigfaltigkeiten von Räumen konstanter Krümmung, PHDThesis, Münster 1995.

[Ku] Kuppe, M., Differentialgeometrie zweidimensionaler singulärer semi-algebraischer Mengen, Diplom Thesis, 1994.

[Mi] Milnor, J., Morse Theory, Annals of Mathematics Studies 51, Princeton University Press 1963.

[Sa] Santalo, L.A., Integral Geometry and Geometric Probability, Reading, Massachusetts, 1976.

[Sch] Scheufler, W., Zweidimensionale semi-algebraische Mannigfaltigkeiten als Aleksandrov Flächen, Diplom Thesis, 1995.

Mathematisches Institut

Einsteinstrasse 62

D-48149 Münster 